Se DivertiR: LeS ENSEIGNEMENTS DE LA BIBLIOTHEQUe D'Une FEMME ARISTOCRATE HONGROISE DE LA FIN DU XVIII ${ }^{\mathrm{E}}$ SIECLE

\title{
Olga Granasztói
}

En Hongrie, au dernier tiers du XVIII et même encore au début du $\mathrm{XIX}^{\mathrm{e}}$ siècle l'accès au divertissement littéraire était particulièrement déterminé par les langues parlées du lecteur. Mais ce n'est absolument pas une question de connaissance de langue qui est en cause: le public des lecteurs hongrois était par nécessité multilingue. Pour de différents usages ils avaient le choix ou étaient obligés de lire des textes en latin, en allemand, en français et en hongrois ou en une autre langue maternelle (slovaque, croate etc.). Choisir une lecture parmi les nouveaux genres populaires répondait à une logique complexe. La littérature de divertissement en langue hongroise était très en retard, par conséquence la part des lecteurs qui choisissaient en nombre des lectures hongroises pour se divertir était minime par rapport à ceux qui choisissaient des livres allemands. Létat de développement de la langue littéraire hongroise était encore peu apte à l'écriture littéraire moderne. Il faut également prendre en considération que la place du divertissement était aussi bien contestée par les littérateurs que les autorités de la censure. Un esprit pragmatique marquait la réflexion littéraire hongroise de l'époque encré dans le principe horatien «d'utile et dulce»: Ce qui divertit doit toujours être utile au sens moral du terme. Quelle littérature répond aux critères du bon divertissement? C'est la question qui préoccupait les gens de lettres en Hongrie, et c'est ce qui a pendant longtemps figé le développement de la littérature écrite en hongrois.

Quant à la librairie, elle devait faire face d'une part à la diversité linguistique du lectorat, d'autres part à la demande croissante pour les nouveaux genres populaires apparaissant très rapidement sur l'horizon du public hongrois. Á la période de transition culturelle des dernières décennies du XVIII ${ }^{e}$ siècle la langue de publication d'un ouvrage divertissant joue un rôle important dans sa réception, ce qu'on peut 
étudier à partir des catalogues de libraires conservés sur le territoire hongrois. On a de la chance qu'ils couvrent une période courte mais très dynamique. ${ }^{1}$ Ces catalogues imprimés entre 1787-1790 contiennent essentiellement des nouveautés de langues étrangères, et la façon dont ils sont structurés montre un certain nombre de similitudes: c'est à partir du milieu des années 1780 que les livres français apparaissent dans quasiment chaque librairie, et qu'ils sont rangés à la fin du catalogue comme une catégorie à part. ${ }^{2}$ Les titres italiens et anglais n'ont pas cette place privilégiée, ils viennent toujours après le français ou mêlés aux français. Le reste est dominé par des titres en allemands et latins, de moins en moins représentatifs, mis souvent dans la catégorie nommée «Auctores classici». Pour mieux orienter le lecteur dans le corpus des nouveautés allemandes - ce qui est fondamentalement différent des catalogues de quelques années plutôt - les libraires procèdent à un classement thématique à l'intérieur de l'ordre alphabétique. Ce classement thématique est une mixture de catégories classiques et de catégories «transitoires» des nouveaux genres littéraires tel que «BriefeLettres», «Geschichte-Histoires» qui renvoient en grande partie à différents types de romans et/ou de nouvelles. Les libraires de Hongrie, quasiment tous d'origine allemande, adoptent ces nouvelles catégories du catalogue de la foire de Leipzig et de catalogues de leur confrères

$1 \quad$ Cf. Olga Granasztor : «Presbourg, Pest, Vienne : réseaux de diffusion de l'imprimé français 1770-1800», Cornova 3 (2/2013), 77-83.

2 Verzeichniss der Bücher welche bey A Löwen, Buchhändler in Pressburg, um beygesetzte Preise verkauft werden. 1788, 1790.; Verzeichniss der neuern Bücher aus allen Theilen der Wissenschaften welche zu haben sind bey Alois Doll und Schwaiger in Pressburg, 1788.; Bücher Verzeichniss aus allen Theilen der Wissenschaften welche bey Philipp Ulrich Mahler Buchändler in Pressburg am beygesetzte Preise verlauft werden. Pressburg, 1788. Erste Fortzetzung von 1788 der Büchers welche bey Philipp Ulrich Mahler in Presburg verkauft werden 1790.; Catalogue des livres françois, italiens et anglois qui se trouvent ches Weingand et Köpff libraires, rue de serpent à Peste, 1781.; Catalogue des livres françois, anglois, italiens qui se trouvent en vente chez Jean-Michel Weingand à Pest et à Ofen, 1789.; Catalogue des livres françois, italiens, anglois et d'autres langues etrangeres, qui se trouvent chez Antoine Loewe, Imprimeur et libraire dans la Rue St. Michel. A Presbourg, 1788. 
allemands. ${ }^{3}$ La situation de la catégorie appelée en allemand «Romane» est la plus révélatrice de ce point de vue: elle reflète ce que le public attendait du genre publié en allemand à cette période là. Ici les titres évoquent essentiellement les différentes facettes du divertissement, et s'efforcent de signaler qu'il s'agit d'un roman. C'est plus par le contenu et moins par des critères formels que l'on identifie le genre dont les frontières restent encore très floues. ${ }^{4}$

Quant aux romans publiés en français et répertoriés dans la rubrique intitulé „livres français", ils reflètent un état plus développé du genre: on a l'impression que le roman en français répond mieux au critères du genre. Dans leur description bibliographique le titre réfère moins au fait qu'il s'agit d'un roman..$^{5}$ Le roman en français ne doit pas se légitimer parce que son public le reconnaît déjà.

Cette différenciation de plus en plus fine des genres littéraires modernes caractérise d'abord la littérature française, ce que la collection des livres français d'une lectrice hongroise du XVIII siècle, passionnée pour la littérature, reflète particulièrement bien. La bibliothèque de la comtesse Julie Csàky manifeste d'une manière extraordinaire le développement de la littérature française moderne du XVIII siècle, son autonomisation par rapport à l'histoire, et son extraordinaire popularité qui gagnait tout le continent jusquà la fin du siècle. Cette bibliothèque offre le panorama complet de ce que la production livresque française ait donné au lectorat européen en matière de lecture contemporaine. Elle est une collection particulière non seulement de par son homogénéité linguistique mais

$3 \quad$ Messkataloge der Frankfurte und leipziger Buchmessen, Ostermesse 1700 Ostermesse 1800. Verfilmung der Ausgabe Leipzig auf Normmicrofiches, hrsg. Bernhard Fabian, Hildersheim, 1979.

4 Par exemple: Carl Gutmann in Halle, kein Roman sondern Wahrheit in Modekleide, Halle, 1786 ; Die Frau Lisel oder die Schöne Nanette. Ein Roman Zum lachen für die Noblesse, u. zum Nachdenken für die Bürger, 1786 ; Uhuhu, oder Hexen-GespensterSchazgräber- und Erscheinungsgeschichten, Erfurt, 1787.

5 P. E.: La Comtesse de Tessan ou l'insuffisance de la Vertu 8, 1785 ; Le mari sentimental, 1785, Genève ; L'Hypocrite démasqué ou Felix et Colomb, Paris, s.d. ; L'amitié dangereuse, Paris, s.d. 
du point de vue de son contenu aussi. ${ }^{6}$ La bibliothèque fut fondée aux alentours de 1770, enrichie jusqu'en 1807-1808, et vendue finalement par la propriétaire, la comtesse Csàky lorsque avec son mari ils décidèrent de mettre fin à leur mariage. La bibliothèque Csáky n'est en effet qu'une partie de la bibliothèque du château de Homonna (aujourd'hui Humenné en Slovaquie), mais une partie bien précise. Il s'agit d'une collection de livres français dont le catalogue in folio, en vélin porte le titre (sur le dos) «Catalogue des livres françoise [sic] et italiens», qui comprend 5160 volumes, répartis en 2330 titres. $^{7}$ Cette collection fut installée dans le château de Homonna, le domaine du comte Csàky, dans la région du nord-est de la Hongrie. Le catalogue est conservé en son intégralité, il n'y manque que la page de titre qui en fut soigneusement coupé. En l'ouvrant, on constate tout de suite qu'on a affaire à un catalogue par matière, et non pas rédigé en ordre alphabétique. Il est divisé en neuf parties distinguées par des titres français: «l. Histoire 2. Philosophie 3. Poésie 4. Poesia in lingua Italiana (avec sept titres seulement) 5. Dictionnaires et voyages 6. Lettres 7. Fables, contes et histoires 8. Romans 9. Mélanges historiques, philosophiques, critiques et amusans.» Selon cette répartition, seule trois matières (philosophie, histoire, poésie) correspondent au classement thématique traditionnel des bibliothèques privées de l'époque. Le reste correspond à un classement des genres de la prose qui rappelle plutôt l'agencement d'un catalogue de librairie. En effet, le contenu de cette collection de livres français ne correspond nullement aux catégories classiques qui inventorient d'habitude un choix encyclopédique des livres. Ici c'est la catégorie classique des belles-lettres qui est surreprésentée et que le propriétaire essaya de trancher en des sous catégories plus ou moins évocatrices. Cette répartition des titres révèle en même temps l'incertitude qui règne encore à cette période-là quant à la définition exacte des genres de la

6 Pour plus de détails sur la comtesse Csáky voir : Olga Granasztor: «Diffusion du livre en français en Hongrie: bilan et perspectives des recherches sur les bibliothèques privées de l'aristocratie (1770-1810) Histoire et civilisation du livre. Revue internationale, tome X, Genève, Librairie Droz, 2014, 200-205.

7 Consultable dans son fonds originel à Arad en Roumanie à la Biblioteca Județeanã «A. D. Xenopol», au département des livres ancien. 


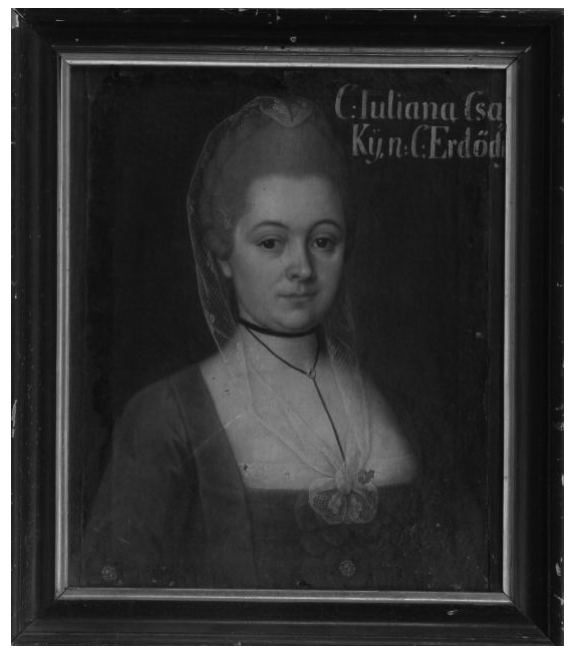

Peintre inconnu: Julianna Csáky née Erdődy, s.d., SNM Muzeum Cerveny Kamen, Slovaquie

prose. Le flottement du genre du roman pèse sur quasi toutes les classes, de livres mentionnés cidessus - autrement dit - bien que les romans soit regroupés dans une classe à part, on en retrouvent dans quasi toutes les autres classes excepté la poésie. Il est impossible d'identifier le dénominateur commun qui distingue clairement l'«histoire» du «roman», le «roman» de la catégorie «conte» ou celle de «mélanges». Les règles du roman n'étant pas encore fixes et figées, le propriétaire inventa un système qui combine des connaissances théoriques préalables et des traits distinctifs occasionnels dont la base est le contenu du livre. Cette effort de ranger un corpus de livres à ce point là moderne est remarquable et reflète le sens littéraire aigu de la comtesse Csàky, qui a non seulement rassemblé ces quelques 5000 volumes, mais a élaboré un système de classement basé sur une connaissance approfondie des livres possédés. Le catalogue, dont la rédaction était dirigée par elle, nous révèle comment cette collection rarissime fut constituée, et combien un propriétaire peut avoir un contact profond avec ses livres chéris. L'intention de collecter de livres français est née vers les années 1770, mais l'idée d'enregistrer les livres dans un catalogue ne fut décidée qu'une vingtaine d'années plus tard. C'est le catalogue lui même qui révèle les étapes du processus de sa constitution. Il fut conçu par trois personnes différentes en quatre étapes. Ces étapes temporaires se révèlent par le changement de l'écriture et de la couleur de l'encre: les différentes écritures signalent les différentes périodes d'inventorier les 




Thomas Ender : The Csáky-Vandernath mansion in Humenné., 1861-63.

Library and Information Centre of the Hungarian Academy of Sciences.

titres qu'on devine par la dernière date de parution dans une période différenciée par l'écriture. La première étape fut ainsi terminée en 1790; la deuxième se distingue par l'écriture d'une autre personne autour de 1792; la troisième n'eut lieu qu'en 1805, et la dernière, par une troisième personne en 1807, juste avant la vente de la collection. Aucune écriture ne ressemble ni à celle de la comtesse ni à celle du comte. Le fait que trois personnes différentes aient été chargées de ce travail confirme que le couple n'avait pas de bibliothécaire attitré. Cependant la comtesse Csàky travaille elle même sur le catalogue avant la vente de la collection: elle corrige systématiquement les fautes d'orthographes françaises commis par les rédacteurs et ajoute de petits remarques, au cas où l'inventaire énumère un ouvrage qui ne fait plus parti de la collection, où qu'il n'est plus complet. Elle signe l'inventaire à la fin de chaque partie, et finalement elle compte le nombre de volumes et de titres qu'elle inscrit avec sa signature à la fin de l'inventaire.

Le catalogue laisse supposer que l'idée de faire une collection à part pour les livres français naît près de vingt ans après les premiers achats. Lorsque la décision est prise de faire une collection séparée et inventoriée, deux tiers des livres français sont déjà acquis. La genèse par 
paliers du catalogue permet de mesurer le rythme des achats, ainsi que l'enrichissement des différentes matières à l'intérieur de la collection pour une période déterminée. Elle est également un indice du changement des centres d'intérêt du collectionneur fortement influencé par les tendances littéraires les plus en vogue. Il faut noter que la rédaction du catalogue est d'autant plus révélatrice qu'elle répertorie les livres au fur et à mesure qu'ils arrivent du relieur à leur place définitive. C'est à dire, le catalogue suit en quelque sorte la chronologie des achats. Il est incontestable que l'élan d'enrichissement de la collection ait atteint son sommet dans les années 1790, puis connut un fort déclin avant la décision d'être vendu en 1808. Le déclin fut le résultat d'une situation financière du couple de plus en plus difficile, mais aussi celui de l'état de santé de la comtesse

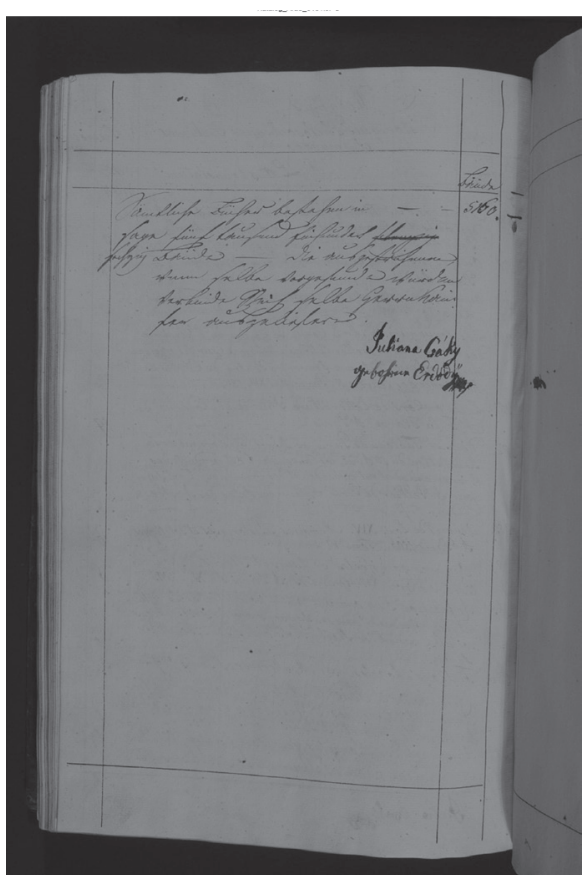

Dernière page du catalogue manuscrit de la bibiliothèque Csàky-Erdődy. Biblioteca Județeanã «A. D. Xenopol » qui avec l'âge, devait renoncer à l'un de ses divertissements préférés que fut la lecture. L'enrichissement de la plus courte période inventoriée permet de se faire une idée du rythme des achats. Entre 1790 et 1792 cent nouveaux titres sont inscrites dans le catalogue, tandis qu'au recensement suivant (qui a eu lieu dix ans plus tard) on ne dénombre au total que deux cents nouveaux titres, ce qui signifie un net recul des achats. Sur toutes les quatre périodes d'enrichissement confondues la matière la plus représentée est celle du roman: on dénombre 567 titres sur les 2330 au total, sans 
compter le nombre de romans distribuer par centaines dans d'autres rubriques. Viennent ensuite les mélanges (383), puis l'histoire (340), la poésie (311), les lettres (288), les fables (191) et enfin la philosophie (178). Ces chiffres, en conséquence du flottement des genres, ne reflètent évidemment qu'une présence proportionnelle des différents types de lectures. Mais malgré cette incertitude on peut quand même détecter des tendances révélant le changement d'intérêt du propriétaire: vers la première décennie du XIX ${ }^{e}$ siècle c'est la philosophie qui recule le plus, tandis que la poésie gagne du terrain. Il n'y rien d'étonnant dans le recul de la philosophie si on prend en considération la perte de popularité de la discipline, suite à la disparition des deux grandes générations de philosophes des Lumières. La poésie par contre peut nous tromper parce que cette catégorie comprend en soi le théâtre, ce qui explique pourquoi cette catégorie peut être aussi populaire dans une période qui n'était pas l'âge d'or de la poésie.

La collection offre un panorama complet de la littérature française du XVIII ${ }^{e}$ siècle, tous genres confondus (théâtre, poésie, conte, roman etc.). Mais elle nous introduit en même temps au monde du livre interdit où selon l'expression des libraires de l'époque - au monde des livres «philosophiques» qui comprennent en soi la littérature libertine, les libelles, les pamphlets politiques, les chroniques scandaleuses, les vies privées, et les classiques de la littérature anticléricale. Ces genres de lecture composent surtout la catégorie «Mélanges», où le trait de caractère commun des titres pourrait être l'esprit d'actualité qui les distingue particulièrement. Il faut également souligner qu'il n'y a pas d'ouvrages édités au XVII ${ }^{e}$ siècle et le nombre de ceux édités dans la première moitié du XVIII est minime par rapport à l'ensemble. Etant donné que le catalogue se constitua au fur et à mesure des achats, c'est à dire les titres sont inscrits dans le catalogue dans une sorte de chronologie, les livres les plus anciens se trouvent toujours dans le premier recensement de 1790. Il semble que la comtesse a jeté les bases de sa bibliothèque française entre les années 1770 et 1790, tous genres confondus. Ce sont les grands classiques qu'elle acheta le premier, ensuite quasi uniquement, 


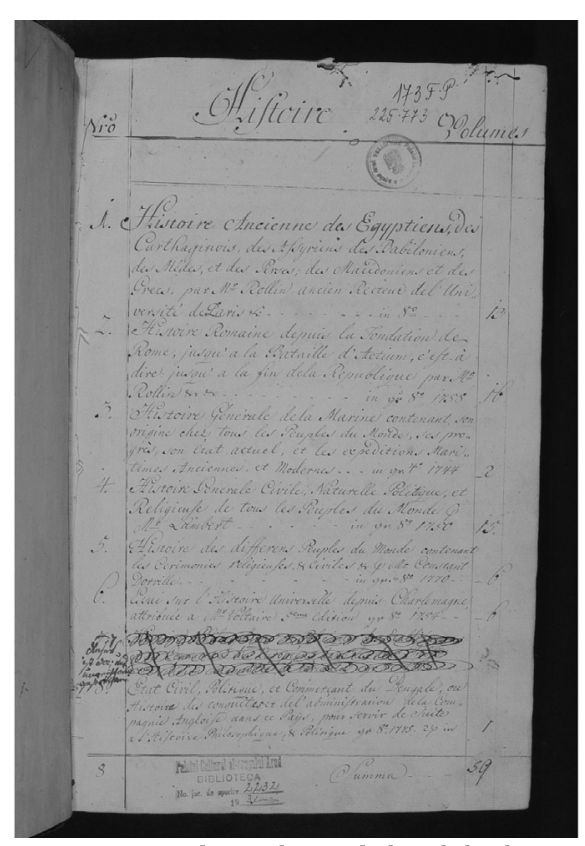

Première page du catalogue de la Bibiliothèque Csáky-Erdődy. Biblioteca Județeanã «A. D. Xenopol»

des nouveautés. La «Philosophie» par exemple commence par l'édition de l'oeuvre complète de Voltaire paru à partir de 1757. Dans la catégorie de «Conte» le $13^{\mathrm{e}}$ titre c'est les Contes de La Fontaine dans une édition de 1703. Dans la catégorie „Lettres” en $6^{\mathrm{e}}$ position on trouve Les Provinciales édité aussi en 1703. L'intérêt de la comtesse, après avoir jetée les bases d'une bibliothèque française contemporaine, et rattrapée son temps, allait essentiellement vers les nouveautés, une tendance qui se dessine nettement à partir du deuxième recensement des livres vers 1792, la période la plus courte dans l'inscription des nouveaux titres. Dans cette courte période de deux années où l'on continue le travail entamé vers 1790, il apparaît que le collectionneur est arrivé à son présent quant à la date de parution des ouvrages achetés: il est vrai que certaines matières ou classes de livres, tel que celles des «Dictionnaires et Voyages», «Philosophie» ou de «Poésie» sont moins exposées à cette soif de nouveautés, étant donné que ce n'est pas dans ces disciplineslà que l'on en produit en grande quantité par rapport à la prose ou la politique. Sur le marché du livre ce n'est pas des ouvrages de géographie ou de philosophie qui apparaissent en nombre dans la décennie approximative de 1792-1805, la décennie où la comtesse ne s'intéresse quasiment qu'aux nouveautés ou à des titres qui manquent encore dans 
sa collection choisie. Vers 1792, dans les catégorie littéraires, telles les „lettres", les «contes et nouvelles», le «roman», et celle de «mélanges», cette synchronie avec l'offre de la libraire française est frappante: parmi «les Lettres» cette tendance est marquée par des titres publiés en 1789 comme La jeune veuve ou Histoire de Cornelia Sedley. Traduit de l'anglois par M. de Saint-Amand, 1789. Parmi les «contes et nouvelles» cette série de nouveautés débute par L'oreille, conte asiatique de Fontette de Sommery publié en 1789. Parmi les «Romans» nous avons Une année de la vie du Chevalier Faublas de Louvet de Couvray publié en 1790. On pourrait continuer l'énumération tant le roman gothique prend une place dominante: Rosa, ou les châteaux en Espagne d'une jeune Angloise, 1790. Lidorie ancienne chronique allusive de Jean Claude Gorjy publié en 1790. Dans la catégorie «Mélanges» c'est les questions d'actualité qui domine comme l'ouvrage intitulé Vies privées des ecclésiastiques, prélats et autres paru en 1791; ou Dénonciations aux français catholiques des moyens employés par l'Assemblée nationale pour detruire en France la religion catholique, 1791.

Trouver une édition plus ancienne est quasi impossible parmi les romans, où à partir de 1792-1793 c'est quasiment un catalogue de librairie d 'assortiment qui s'étale devant nos yeux. Le roman de divertissement règne à partir de cette date, comptant 132 nouveaux titres répertoriés jusquà 1807. Un léger changement de l'intérêt de la comtesse apparaît parallèlement à la chute du nombre des titres des dernières années. Exceptée la classe du roman, des éditions plus anciennes apparaissent parmi les toutes dernières entrées dans la catégorie de «Lettres», où l'on retrouve les ouvrages de Restif de la Bretonne en série: La Mimographe, L'Andrographe (1782) et Les Gynographes (1777). En philosophie l'écart entre la date de parution et celle de l'inscripition dans le catalogue est éclatant: De la République et du gouvernement de Ciceron (1788). La République de Platon ou du juste et de l'injuste (1787). Les entretiens mémorables de Socrate traduit par Levesque (1786). Enfin, les deux derniers titres inscrits parmi les «Contes et Nouvelles» sont très parlants: comme si la sagesse gagnait sur la légèreté et la frivolité: Contes non immoraux de Rigaud de Montmeyant (1806) ou Les matinées du hameau ou contes d'un grand père à 


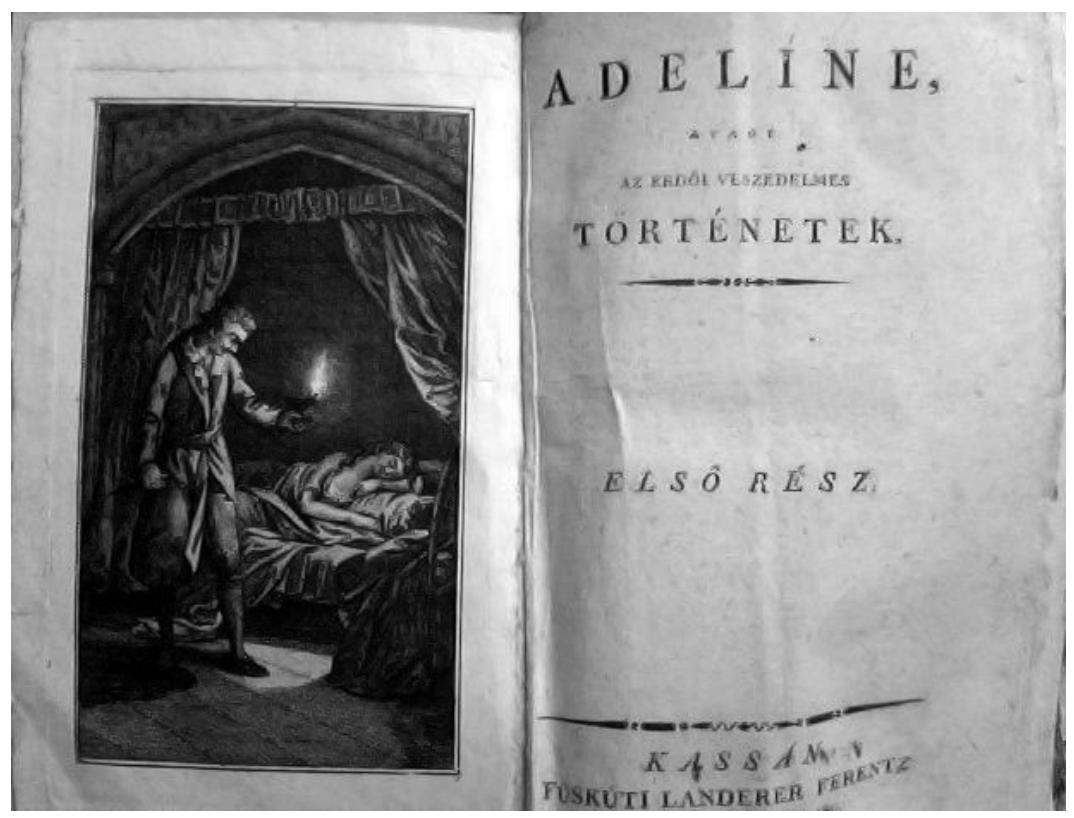

Adeline, avagy az erdői veszedelmes történetek [Ann Radcliffe, The Romance of the Forest./Adeline oder die Abentheuer im Walde] Kassa, 1801.

ses petits enfants (1804). Ces deux titres reflètent une sorte de changement de lecture généré par l'âge du collectionneur. En effet, la comtesse avait déjà plus de soixante ans lorsqu'elle s'est séparée de sa collection chérie. La collection de la comtesse Csàky est tout à fait exceptionnelle même à l'échelle européenne tant elle représente un choix de lecture à la mode, un soif d'actualité que le marché bien organisé du livre français satisfaisait avec succès jusqu'au tournant du siècle. La vente de cette collection tombe sur la période, le début des années 1800, où les pratiques de lecture changent. La littérature de divertissement écrite en français connaît un recul marquant dans cette partie d'Europe. En Hongrie ce n'est plus les lectures françaises qu'on cherche, mais les nouveautés publiées en allemand. C'est l'allemand qui prend la place du français en tant que langue véhiculaire aussi pour faire connaître au public le 
roman anglais qui domine cette période, et qui est traduit, adapté où imité en allemand dans les centres de traductions qu'on appelle aussi des «usines» de traductions. Cette filiation est d'autant plus importante qu'elle conduit à la naissance du roman de divertissement en hongrois sous l'influence du roman anglais interprété en allemand. C'est ainsi qu'on publie dans la toute première collection hongroise de romans, intitulé «collection rose», le roman d'Ann Radcliffe The Romance of the Forest, paru en anglais en 1791, traduit en allemand en 1793 sous le titre Adeline oder die Abentheuer im Walde, puis traduit en hongrois à partir de la version allemand en 1801 sous le même titre Adeline, avagy az erdői veszedelmes történetek. Ce roman gothique plutôt sombre et angoissant est tout sauf rose. En autorisant sa parution, la censure et les théoriciens de la lecture pour femmes semblent s'être adaptés à ce nouveau monde du livre, essentiellement dominé par l'offre et la demande.

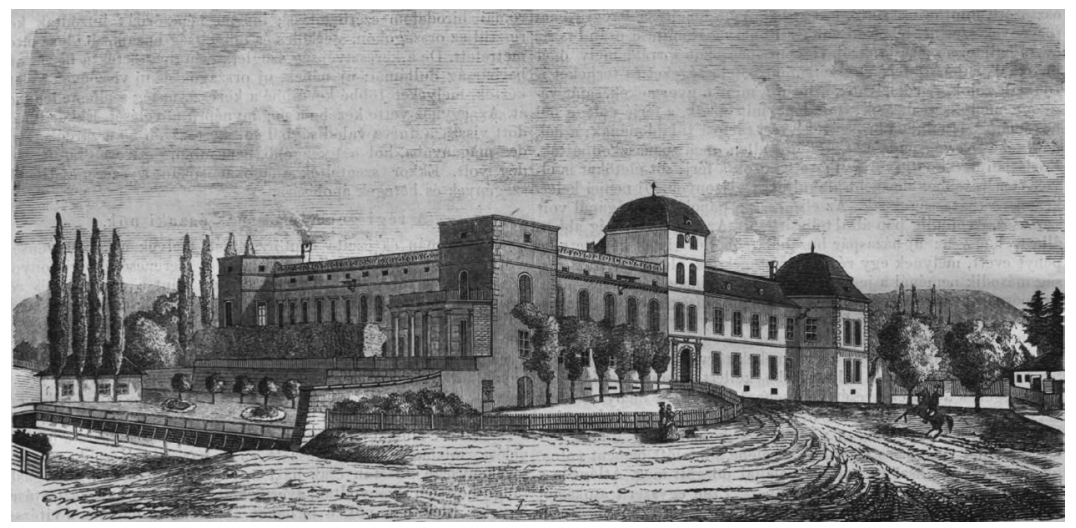

The Csáky-Vandernath mansion in Humenné. In : Vasárnapi Újság, 1861. 
LES BIBLIOTHÈQUES ET L'ÉCONOMIE DES CONNAISSANCES Bibliotheken und die ÖKonomie des Wissens

$$
145^{\circ}-185^{\circ}
$$

Colloque international - Internationale Tagung 9-13 avril/April 2019 Sárospatak (Hongrie/Ungarn)

Édité par

Frédéric Barbier, István Monok et Andrea Seidler 


\section{L'Europe en réseaux}

Contribution à l'histoire de la culture écrite 1650-1918

Vernetztes Europa

Beiträge zur Kulturgeschichte des Buchwesens 1650-1918

Édité par

Frédéric Barbier, Marie-Elisabeth Ducreux, Matthias Middell, István Monok, Éva Ringh, Martin Svatoš

Volume VIII

École pratique des hautes études, Paris

École des hautes études en sciences sociales, Paris

Centre des hautes études, Leipzig

Bibliothèque nationale Széchényi, Budapest

Bibliothèque et centre d'information de l'Académie hongroise des sciences, Budapest 
LeS BIBLIOTHÈQUeS ET L'ÉCONOMIE DES CONNAISSANCES BibliotheKen und die ÖKonomie des Wissens

$$
145^{\circ}-185^{\circ}
$$

Colloque international - Internationale Tagung 9-13 avril/April 2019 Sárospatak (Hongrie/Ungarn)

Édité par

Frédéric Barbier, István Monok et Andrea Seidler

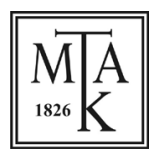

Magyar Tudományos Akadémia Könyvtár és Információs Központ Budapest 2020 
Mise en page

Viktória Vas

ISBN 978-963-7451-57-7

DOI 10.36820/SAROSPATAK.2020 
Préface

István MoNOK

Bibliothecae mutantur - Quare, quemadmodum et quid attinet?

Transformations de la composition thématique

des bibliothèques du Royaume de Hongrie aux $\mathrm{XV}^{\mathrm{E}}-\mathrm{XVI} \mathrm{I}^{\mathrm{e}}$ siècles....11

Marianne CARbonnier-Burkard

Les bibliothèques des Églises réformées françaises au XVII siècle.... 30

Max Engammare

De la bibliothèque de l'Académie de Calvin (1570) a la bibliothèque de l'Académie de Bèze (1612) à travers leur catalogue: Continuités et ruptures jusqu'au troisième catalogue de 1620........... 57

Róbert OLÁ́

Obsolescent Reformed Libraries in the seventeenth and eighteenth Century Carpathian Basin

Ádám Hegri

Moderner Zeitgeist - veraltete Lesestoffe. Bibliotheken reformierten Pfarrer um die Wende des 18. zum

19. Jahrhunderts im Königreich Ungarn

Petr MAšEK

Zierotin Library in Velké Losiny in Sixteenth and Seventeenth century. 136

Detlef HABERLAND

Schlesische Bibliotheken Zeichen der intellektuellen Vielfalt einer zentralen Bildungsregion in Europa 146

Thomas WaLLNIG

Sebastian Tengnagel und Johann Seyfried - Österreichische

Geschichtsschreibung zwischen Späthumanismus und

Gegenreformation 
Elisabeth EngL-Ursula RautenBerg

Christoph Jacob Trew - Bibliothek und Sammeln in der Gelehrtengemeinschaft der ersten Hälfte des 18. Jahrhunderts.

Helwi BLOM

Philosophie ou Commerce?

L'évolution des systèmes de classement bibliographique dans les catalogues de bibliothèques privées publies en France au XVIII ${ }^{\mathrm{e}}$ siècle.

Maria Luisa López-VIDrIero Abelló

Les meubles de la connaissance: façons de devenir sage à prix fixe.

Frédéric BARbier

Distinction, récréation, identité: la trajectoire des « romans» en France sous d'Ancien Régime.

\section{Andrea SeIdler}

Die praktische Bedeutung ungarischer Sammlungen und Bibliotheken für führende Gelehrte des Königreichs Ungarn im späten 18. Jahrhundert am Beispiel des Jesuiten Georg Pray (1723-1801).

\section{Olga Granasztói}

Se divertir: les enseignements de la bibliothèque d'une femme aristocrate hongroise à la fin du XVIII siècle

Christophe Didier

La naissance du théâtre „des boulevards”, ou Comment la banlieue entre en bibliothèque (1780-1830).

\section{Andrea De Pasquale}

La nascita delle riserve di libri antichi in Italia

Index des noms de personne et de lieu..... 